\section{Pancreatic Extramedullary Plasmacytoma Presenting as a Pancreatic Mass}

\author{
Michelle Le*, Balarama Krishna Surapaneni*, Vardhmaan Jain, \\ Rakesh Vinayek and Sudhir K Dutta
}

Department of Gastroenterology, Sinai Hospital, Baltimore, MD, USA.
Clinical Medicine Insights: Gastroenterology Volume 11: 1-5

(C) The Author(s) 2018

Article reuse guidelines:

sagepub com/journals-permissions

DOI: $10.1177 / 1179552218801603$

(S)SAGE

\begin{abstract}
Extramedullary plasmacytomas (EMP) are a subcategory of plasma cell neoplasm that involves organs outside the bone marrow. Involvement of the pancreas is relatively rare, reported in only $2.3 \%$ of autopsies. Radiologic findings in plasmacytoma are nonspecific, but endoscopic ultrasound fine-needle aspiration is a fast and reliable technique to acquire a histologic sample for initial diagnosis. Recently, the use of fluorine-18 fluorodeoxyglucose PET/CT has been recommended in patients with active multiple myeloma and solitary plasmacytoma. We present an interesting case of primary EMP in the pancreatic body encasing the portal vein as well as the celiac artery, which was detected before the patient was diagnosed of multiple myeloma.
\end{abstract}

KEYWORDS: Multiple myeloma, pancreatic mass, extramedullary plasmacytoma

RECEIVED: February 26, 2018. ACCEPTED: August 29, 2018

TYPE: Case Report

FUNDING: The author(s) received no financial support for the research, authorship, and/or publication of this article.
DECLARATION OF CONFLICTING INTERESTS: The author(s) declared no potentia conflicts of interest with respect to the research, authorship, and/or publication of this article.

CORRESPONDING AUTHOR: Balarama Krishna Surapaneni, Department of Gastroenterology, Sinai Hospital, 2401 W Belvedere Avenue, Baltimore, MD 21215, USA. Email: balaram_s1990@yahoo.com

\section{Introduction}

Extramedullary plasmacytoma (EMP) is characterized by localized monoclonal plasma cell proliferation that forms a solitary lesion outside the bone marrow. It is usually diagnosed in patients with widespread multiple myeloma (MM). Extramedullary plasmacytoma in its primary form is extremely rare. They are commonly found in the head and neck region (80\%) with only $5 \%$ to $10 \%$ of solitary cases arising from the gastrointestinal (GI) tract. The GI involvement is most common in the small intestine followed by the stomach. ${ }^{1}$ We present a rare case of pancreatic EMP in a patient with newly diagnosed advance MM.

\section{Case Summary}

A 73-year-old woman with no significant past medical history presented to the emergency department (ED) with a history of left scapular and bilateral rib pain on October 2015. X-ray imaging was unremarkable, and the patient was discharged home with ibuprofen. After 2 months, the patient saw her family physician and complained of decrease appetite, worsening periumbilical discomfort and a pulsatile mass in her abdomen. She was found to be anemic with hemoglobin of $9.5 \mathrm{~g} / \mathrm{dL}$, hematocrit of $29.4 \%$, and platelet of $123000 / \mathrm{mm}^{3}$. Electrolyte profile showed borderline hypokalemia (potassium levels of $3.3 \mathrm{mg}$ / $\mathrm{dL}$ and normal calcium levels $(9.5 \mathrm{mg} / \mathrm{dL})$. Kidney function tests showed borderline elevated urea levels $(=24 \mathrm{mg} / \mathrm{dL})$, a normal creatinine level $(0.93 \mathrm{mg} / \mathrm{dL})$ and serum albumin were found to be $4.0 \mathrm{mg} / \mathrm{dL}$. An ultrasound of the abdomen showed a pancreatic mass and a follow-up computed tomographic (CT) scan of the abdomen and pelvis revealed a large pancreatic body mass encasing the portal vein as well as the celiac artery (Figure

*Both the authors are co-first authors.
1). Endoscopic ultrasound (EUS) revealed a well-defined round mass arising from the body, measuring $6 \mathrm{~cm}$ on the long axis and $4 \mathrm{~cm}$ on the short axis (Figure 2). No peripancreatic lymphadenopathy was noted. The fine-needle aspiration (FNA) biopsy was only suspicious for atypical plasma cells.

A week later, she underwent CT-guided core biopsy of the pancreatic mass at the discretion of her oncologist for further immunochemistry testing as the FNA biopsy only stated "suspicious" for atypical plasma cells. Computed tomographyguided biopsy of the pancreatic mass was remarkable for atypical plasma cells with positive CD138 (Figure 3). They also expressed CD56 as well as CD20. In situ hybridization suggested that the plasma cells expressed both kappa and lambda light chains. The patient was thought to have a plasma cell dyscrasia. She underwent a bone marrow biopsy which revealed mildly hypercellular marrow and 50\% to $70 \%$ plasma cell (major criteria; Figure 4). An aspirate was remarkable for 34\% plasma cells which were CD38 positive by flow cytometry. They were monotypic demonstrating lambda chain (Figure 5). Immunoglobulin studies revealed an $\mathrm{IgG}$, which was elevated, IgM less than 21, IgA less than 8 (minor criteria), and $\beta_{2}$ microglobulin was 7.2. There was also a monoclonal spike notes. She was diagnosed with MM according to the modified criteria. Positron emission tomography/computed tomography $(\mathrm{PET} / \mathrm{CT})$ scan revealed a pancreatic mass with focal intense uptake and a hypermetabolic soft tissue mass in the ventral right abdomen. There were multiple skeletal metastasis involving right mandibular ramus, the $\mathrm{C} 6$ vertebrae, and lytic lesions in the transverse process of T3 and numerous lesions within the ribs. There was also focal uptake in the 5 th and 7 th thoracic spine vertebral bodies, the right posterior superior iliac crest, the mid distal left sacrum, and proximal femurs. The patient underwent chemotherapy treatment with 


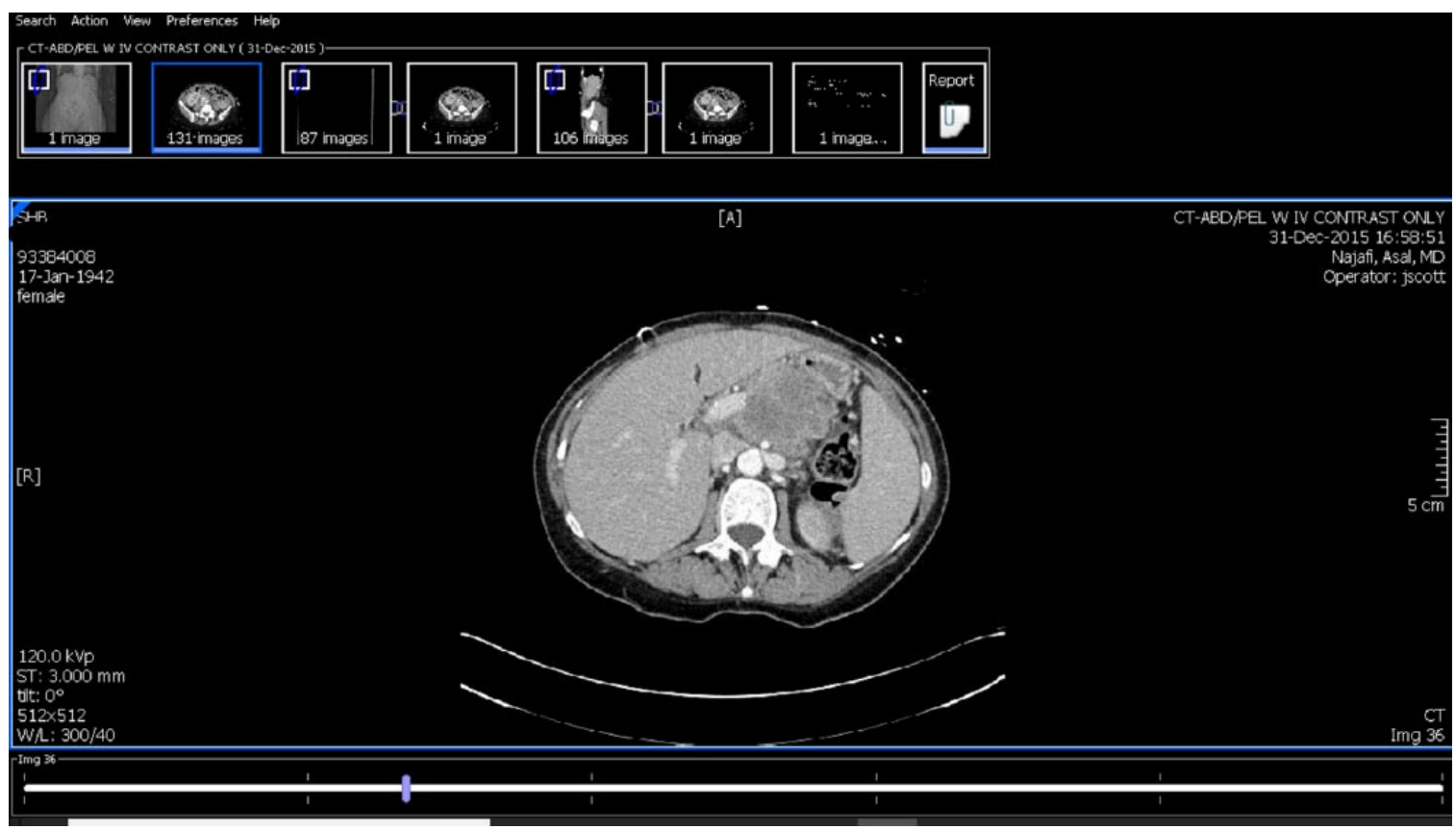

Figure 1. Computed tomographic scan of abdomen with contrast-large pancreatic body mass encasing the portal vein as well as the celiac artery.
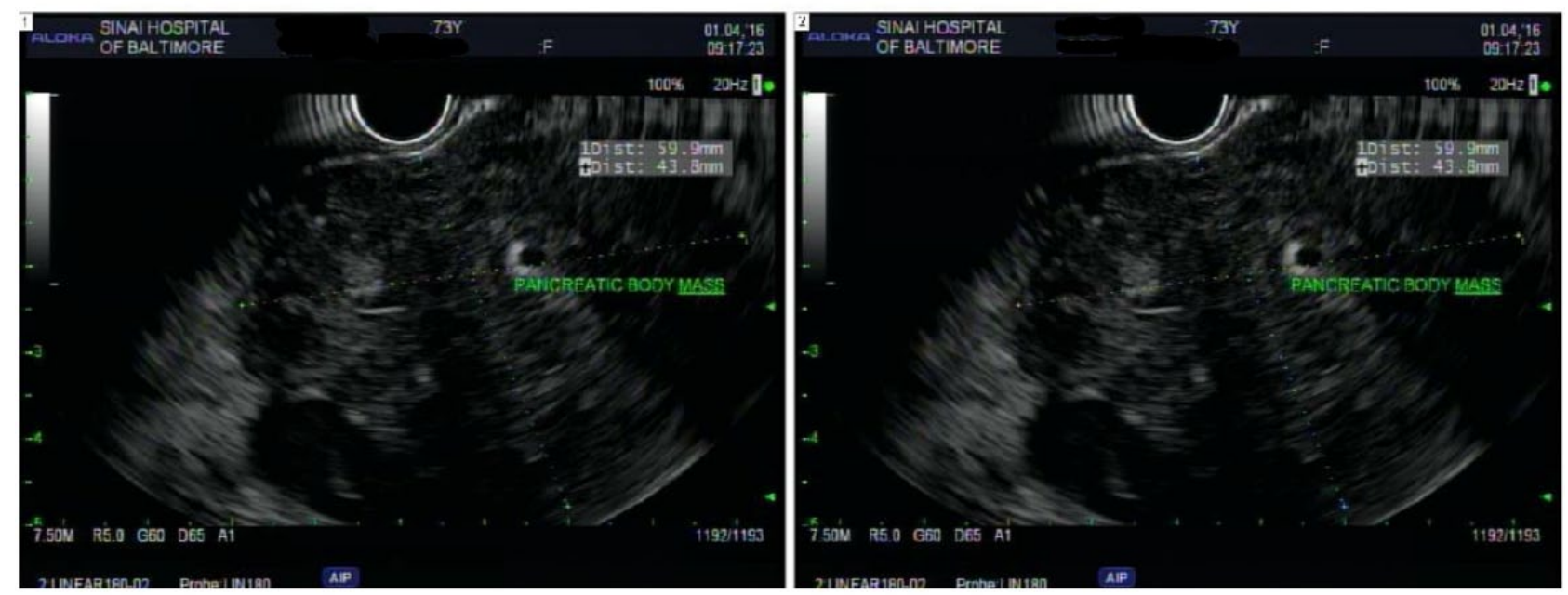

\section{Pancreatic body mass}

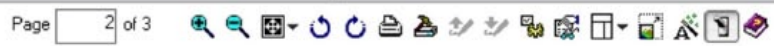

Figure 2. Endoscopic ultrasound-well-defined mass arising from the pancreas. Mass on ultrasonography was hypoechoic and heterogeneous. Mass measured $6 \mathrm{~cm}$ on long axis and $4 \mathrm{~cm}$ on short axis. The mass was encasing the celiac artery.

lenalidomide, bortezomib, and dexamethasone (RVD) along with elotuzumab and responded nicely but had mild peripheral neuropathy

After 2 weeks, she underwent a magnetic resonance imaging which showed diffuse moderate infiltration by a small leftsided extradural mass at T1-weighted imaging with no significant neural compression. There was also a pathologic compression fracture with posterior bony retropulsion involving $\mathrm{C} 6$ and associated mild cord compression. She was then initiated on Cytoxan, carfilzomib, and Decadron and received a brief course of radiotherapy between cycles 1 and 2. Then, she was started on daratumumab and pomalidomide and subsequently Cytoxan was added. During this time, she received palliative abdominal radiation.

After 9 months, the patient later developed a liver mass and underwent a CT scan of abdomen without contrast. Report showed hepatomegaly with multiple nodular masses in liver. Due to the rare nature of $\mathrm{MM}$ arising as nodular liver lesions, 


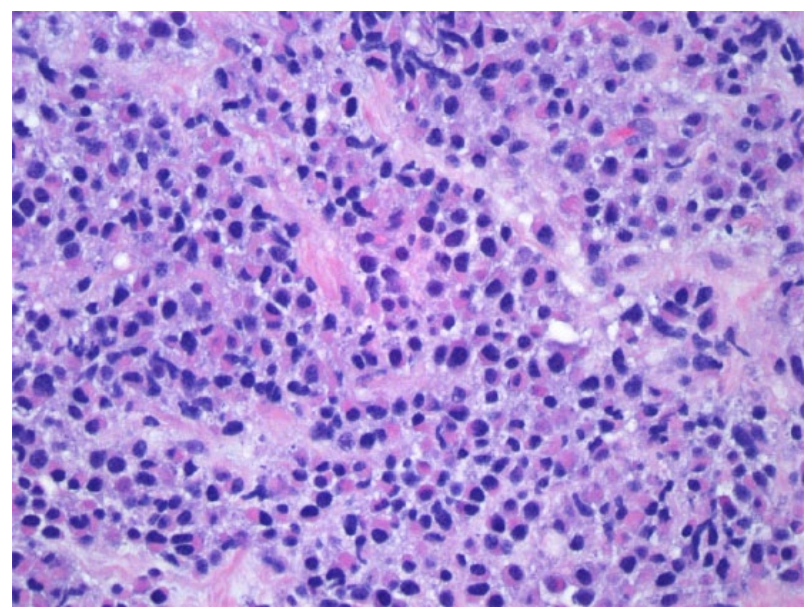

Figure 3. Pancreatic biopsy shows predominant population of atypical plasma cells that are CD138 positive: Hematoxylin and Eosin staining, magnification $\times 400$.

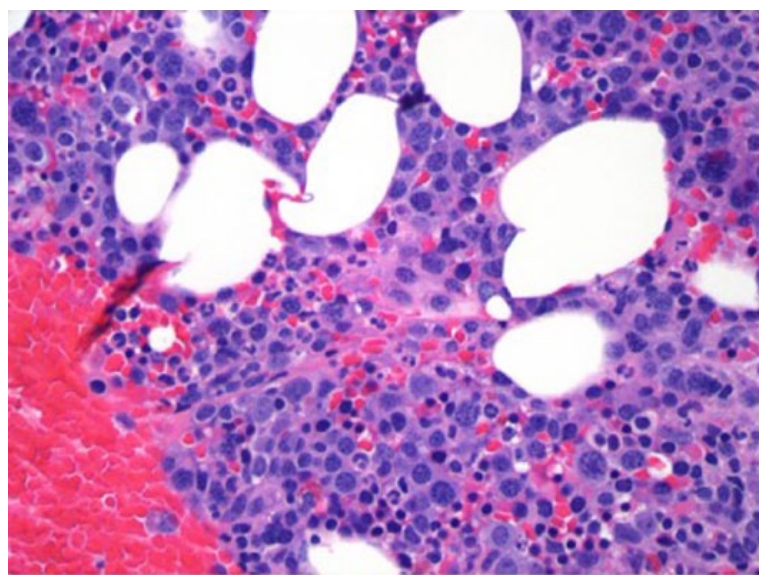

Figure 4. Bone marrow biopsy shows a mildly hypercellular marrow averaging $50 \%$ to $60 \%$. Cellularity is primarily due to increase in plasma cells which are diffusely distributed throughout the marrow space and account for approximately $50 \%$ of the nucleated elements: Hematoxylin and Eosin staining.

a liver biopsy was ordered to rule a second primary neoplasm. The biopsy results revealed plasmacytoma with progression to stage 3 metastasis MM (Figures 6 to 8). She was actively receiving chemotherapy and discharged. She later presented to the ED 3 weeks later with altered mental status and fever. Her blood pressure (BP) was 90/50 with heart rate of 138 with a low oxygen saturation of $90 \%$ on room air. Her respiratory rate was in 30 seconds and she was using accessory muscles of respiration with a paradoxical breathing pattern. The patient was intubated in ED and transferred to intensive care unit. She was started on intravenous fluids and intravenous antibiotics (Zosyn, Vancomycin, and tobramycin) for suspected sepsis. Her white blood cell count was 0.77 , international normalized ratio of 2.0, platelet count 21000 (thrombocytopenia), and lactate was $4.8 \mathrm{mg} / \mathrm{dL}$. Chest X-ray revealed bilateral lower lobe infiltrates likely source of sepsis. Later, her lactate levels rose to $5.7 \mathrm{mg} / \mathrm{dL}$ and a bicarbonate drip was initiated for refractory acidosis. Hemoglobin and hematocrit dropped to 4.1 and 12.3,

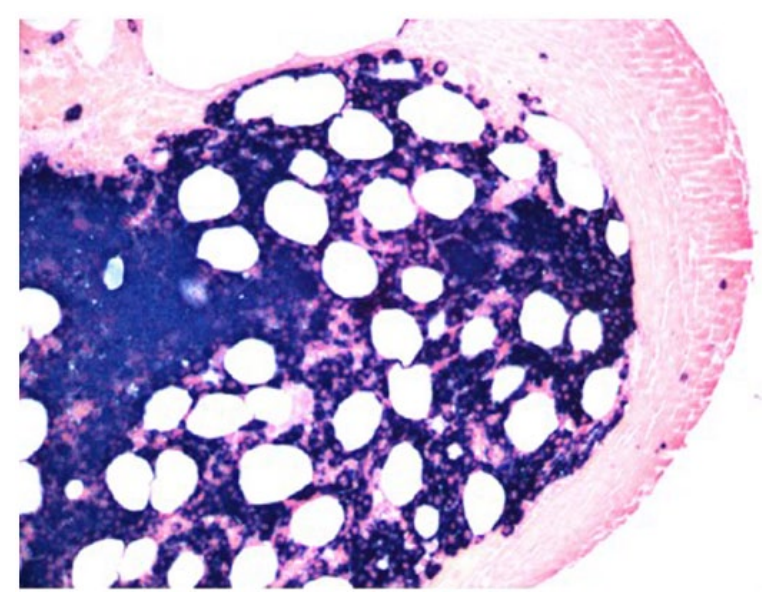

Figure 5. Bone marrow, flow cytometric evaluation shows monotypic lambda light chain-positive plasma cell population: Hematoxylin and Eosin staining.

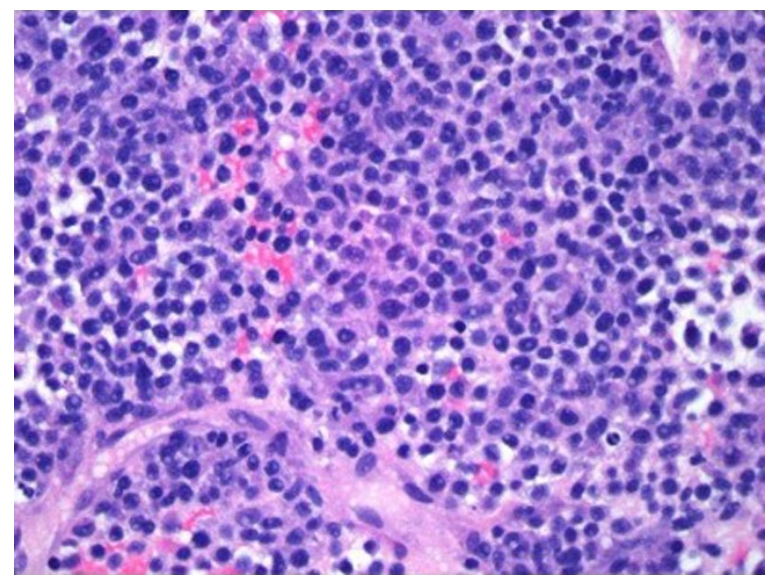

Figure 6. Liver biopsy shows malignant neoplasia that is poorly differentiated but focally has plasmacytic features: Hematoxylin and Eosin staining, magnification x 400 .

respectively, with platelets further worsening to 11000 . Two units of blood transfusion and 1 pack of platelets were ordered. Her BP further worsened to 70/20 and Levophed was increased to maximum dose. Vasopressin drip was started but did not improve her hypotension; hence, epinephrine drip was started. All 3 vasopressors reached maximum doses but her BP failed to improve. The family was informed and they opted a do not resuscitate (DNR). The general condition of the patient worsened and eventually died due to septic shock.

Ethical approval was not required for this case report as it did not relate to patient's privacy or treatment. The informed consent for description of the case report could not be obtained because the patient was dead. However, consent from the spouse was taken.

\section{Discussion}

We have described a rare case of pancreatic plasmacytoma without symptoms of MM. Plasmacytoma can be primary or secondary with secondary form more common. The most commonly involved sites are the liver, spleen, and lymph nodes. 


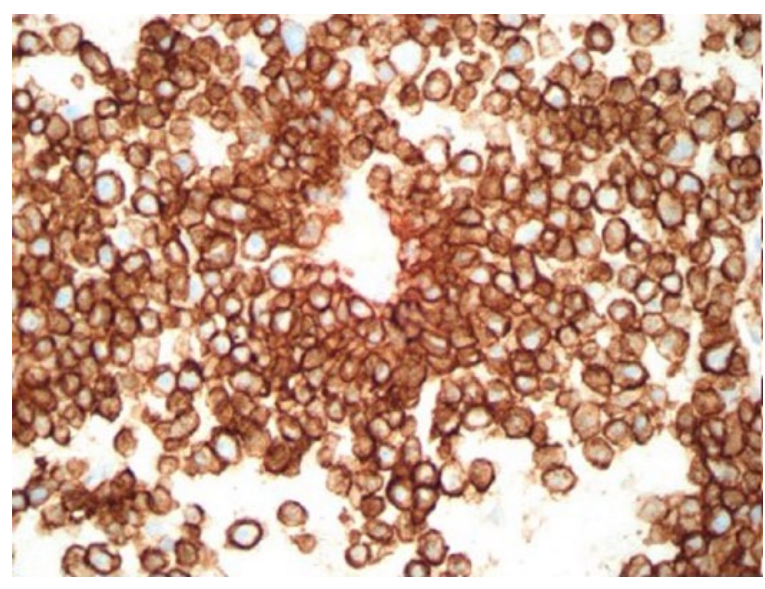

Figure 7. Liver biopsy immunostain CD138: diffuse strong positive.

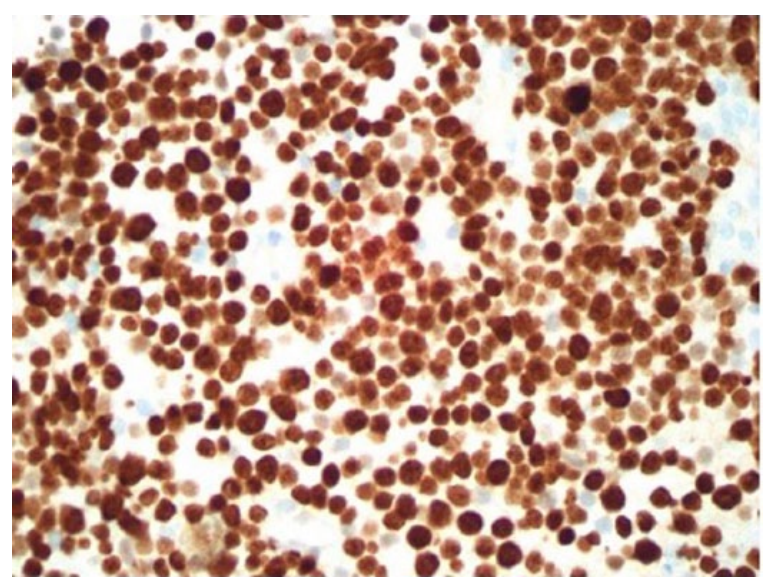

Figure 8. Liver biopsy immunostain Ki67: high proliferation rate approaching $100 \%$.

Involvement of the pancreas is relatively rare, reported in only 2.3\% of autopsies. ${ }^{2}$ Overall, the occurrence of EMPs has been reported to be associated with poor prognosis, high mortality rate, and a shorter overall survival time in patients with MM. Favorable outcomes in cases of EMP have been linked to tumor size $<4 \mathrm{~cm}$, age $<50$ years, patients with head and neck EMP, and serum $M$ protein negativity. All these prognostic factors were absent in our patient pointing toward an adverse outcome. $^{3}$

Our patient was diagnosed as primary EMP because the diagnosis of plasmacytoma was made before the diagnosis of MM. To date, there are more than 63 cases in the English literature of EMP with pancreatic involvement. ${ }^{4,5}$ Although they can develop in any part of the pancreas, many of these lesions are in the pancreatic head. The patient described in our case report has a large pancreatic body mass encasing the portal vein as well as the celiac artery which is even rarer in presentation. She later went on to develop mass in liver with advanced stage 3 prognosis.

Radiologic findings in plasmacytoma are nonspecific, but EUS-FNA is a fast and reliable technique to acquire a histologic sample for initial diagnosis. ${ }^{6}$ However, immunohistochemical analysis of the biopsy specimen or flow cytometry of the aspirated material is crucial to prove the monoclonality and the final diagnosis of a plasma cell neoplasm. ${ }^{2}$ Recently, the use of 18FD-FDG PET/CT has been recommended in patients with active $\mathrm{MM}$ and solitary plasmacytoma. ${ }^{7}$ 18FD-FDG $\mathrm{PET} / \mathrm{CT}$ combines functional imaging provided by PET with morphologic evaluation assessed by CT, to detect metabolically active plasma cells both inside and outside the bone marrow with anatomical localization, size and metabolic properties of focal lesions, or EMP, or both to predict patients' clinical outcome and to assess therapy-induced changes in tumor-cell metabolism. ${ }^{7}$ Plasma cell tumors tend to be radiosensitive and chemosensitive so the treatment of choice for EMPs is generally some combination of surgery, chemotherapy, and radiation therapy. Other methods of treatment under investigation are thalidomide, angiogenic agents, and stem cell transplant for MM. ${ }^{8}$ Newer molecular targets for targeted therapy are being evaluated and experience with chimeric antigen receptor T-cell targets including BCMA (B-cell maturation antigen), CD19, KLC, CD138 has shown nearly 100\% global response in patients with advanced MM. ${ }^{9}$

In summary, we have presented a rare case of primary EMP in the pancreatic body encasing the portal vein as well as the celiac artery which was detected before the diagnosis of MM. Our case was one of the high-grade EMPs that was managed by the currently recommended chemo-radiation therapy guidelines without a favorable outcome thus pointing the need for further studies evaluating adjuvant treatment regimens (based on novel agents) for these high-risk patients. ${ }^{10}$ Even though it is a rare entity, it should be considered as a differential diagnosis in patients presenting with bone pain and radiologic findings with a pancreatic body mass and multiple liver masses. This case illustrates a rare metastasis of MM to the pancreas with involvement of portal vein and celiac artery. It also emphasizes the low morbidity of current biopsy techniques.

\section{Author Contributions}

ML involved in drafting the manuscript or revising it critically for important intellectual content. BKS involved in drafting the manuscript or revising it critically for important intellectual content and agreed to be accountable for all aspects of the work in ensuring that questions related to the accuracy or integrity of any part of the work are appropriately investigated and resolved. Involved in lierature search and final draft from start to finish. VJ involved in drafting the manuscript or revising it critically for important intellectual content. RV is involved in substantial contributions to conception and design of case report. SD agreed to be accountable for all aspects of the work in ensuring that questions related to the accuracy or integrity of any part of the work are appropriately investigated and resolved, gave final approval of the version to be published. 


\section{REFERENCES}

1. Gohil MH, Bhavsar DC, Suryanarayana U, Jetly DH. Plasmacytoma rectum extending to para-rectal region. J Cancer Res Ther. 2015;11:662. https:// doi.org/10.4103/0973-1482.140799.

2. Da Silva RL. Pancreatic involvement by plasma cell neoplasms. J Gastrointest Cancer. 2012;43:157-167. https://doi.org/10.1007/s12029-011-9314-9.

3. Wen G,WangW,ZhangY,Niu S,LiQ, LiY.Management of extramedullaryplasmacytoma: role of radiotherapy and prognostic factor analysis in 55 patients. Chin J Cancer Res. 2017;29:438-446. https://doi.org/10.21147/j.issn.1000-9604.2017.05.08.

4. Williet N, Kassir R, Cuilleron M, et al. Difficult endoscopic diagnosis of a pancreatic plasmacytoma: case report and review of literature. World J Clin Oncol. 2017;8:91-95. doi:10.5306/wjco.v8.i1.91

5. $\mathrm{Lu} \mathrm{T,} \mathrm{Pu} \mathrm{H,} \mathrm{Zhao} \mathrm{G.} \mathrm{Primary} \mathrm{pancreatic} \mathrm{plasmacytoma:} \mathrm{a} \mathrm{rare} \mathrm{case} \mathrm{report.} B M C$ Gastroenterol. 2017;17:167.
6. Akyuz F. Rare pancreas tumor mimicking adenocarcinoma: extramedullary plasmacytoma. World J Gastrointest Endosc. 2014;6:99-100. https://doi.org/ 10.4253/wjge.v6.i3.99.

7. Cavo M, Terpos E, Nanni C, et al. Role of ${ }^{18} \mathrm{~F}-\mathrm{FDG}$ PET/CT in the diagnosis and management of multiple myeloma and other plasma cell disorders: a consensus statement by the International Myeloma Working Group. Lancet Oncol. 2017;18:e206-e217. https://doi.org/10.1016/S1470-2045(17)30189-4.

8. Hatem M, So B, Gray R, Wong J. Plasmocytoma presented as pancreatic head mass. Radiol Case Rep. 2015;10:81-87. https://doi.org/10.1016/j.radcr.2015.06.008.

9. Sohail A, Mushtaq A, Iftikhar A, et al. Emerging immune targets for the treatment of multiple myeloma. Immunotherapy. 2018;10:265-282. https:// doi.org/10.2217/imt-2017-0136.

10. Caers J, Paiva B, Zamagni E, et al. Diagnosis, treatment, and response assessment in solitary plasmacytoma: updated recommendations from a European Expert Panel. J Hematol Oncol. 2018;11:10. https://doi.org/10.1186/s13045-017-0549-1. 